http://jmscr.igmpublication.org/home/ ISSN (e)-2347-176x ISSN (p) 2455-0450

crossref DOI: https://dx.doi.org/10.18535/jmscr/v7i12.12

\title{
Clinico -epidemiological Study of Ocular Rosacea: A Retrospective Study
}

\author{
Authors \\ Dr Arti Sareen ${ }^{1}$, Dr Mudita Gupta ${ }^{2}$ \\ ${ }^{1}$ Medical Officer, DDUZH, Shimla \\ ${ }^{2}$ Assistant Professor, Department of Dermatology, IGMC, Shimla
}

\begin{abstract}
Background: Rosacea is a relatively common dermatosis which is characterized by erythema, telangiectasias, papules, and pustules in the centro-facial area.

Aims: To study the clinico- epidemiological profile of ocular rosacea.

Methods: Records of patients of rosacea were screened. Eye symptoms and signs of all patients with either ocular or cutaneous rosacea were looked for. The demographics and the clinical pattern of these patients were recorded
\end{abstract}

Results: A total of 100 patients of rosacea were screened. The female and male ratio was 8:1. There were

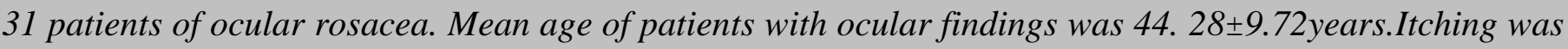
the most common (80.64\%) presenting symptom and lid margin irregularity(58.06\%) commonest eye sign followed by meibomian gland dysfunction(54.83\%).

Conclusion: Ocular rosacea is not an uncommon disease. Clinical manifestations of ocular rosacea may be trivial leading to under diagnosis. Early diagnosis and treatment may prevent the discomfort and the deterioration of vision.

Keywords: Rosacea, Ocular, under diagnosed.

\section{Introduction}

Rosacea is a chronic inflammatory disease which is more common in middle aged females. Cutaneous manifestations in the form of telangiectasias, papules or pustules are usually seen. Ocular involvement in rosacea is also seen but is often overlooked. Ocular involvement is seen in $6-72 \%$ of patients with rosacea. ${ }^{[1]}$ Rosacea can involve different structures of eye e.g, meibomian gland, conjunctiva or cornea. About one third of patients of rosacea can involve the cornea which can be sight threatening. ${ }^{[2]}$

\section{Material and Methods}

This was a retrospective study where records of 100 patients of rosacea in the last 6 months attending ophthalmology OPD and dermatology OPD were screened. Patients who had any complaints or signs suggesting eye involvement were observed. These findings were recorded and demographic profile of such patients were observed. Statistical analysis was done using epi info7.2.2 All discrete variables were recorded as frequency and continuous variables as means, medians and standard deviation. 


\section{Results}

We screened records of 100 patients of rosacea.

There were 89 females and 11 males Ocular involvement was seen in 31 patients. Out of these 28 were females (31.4\% of total females) and 3 males $(27.2 \%$ of total males).

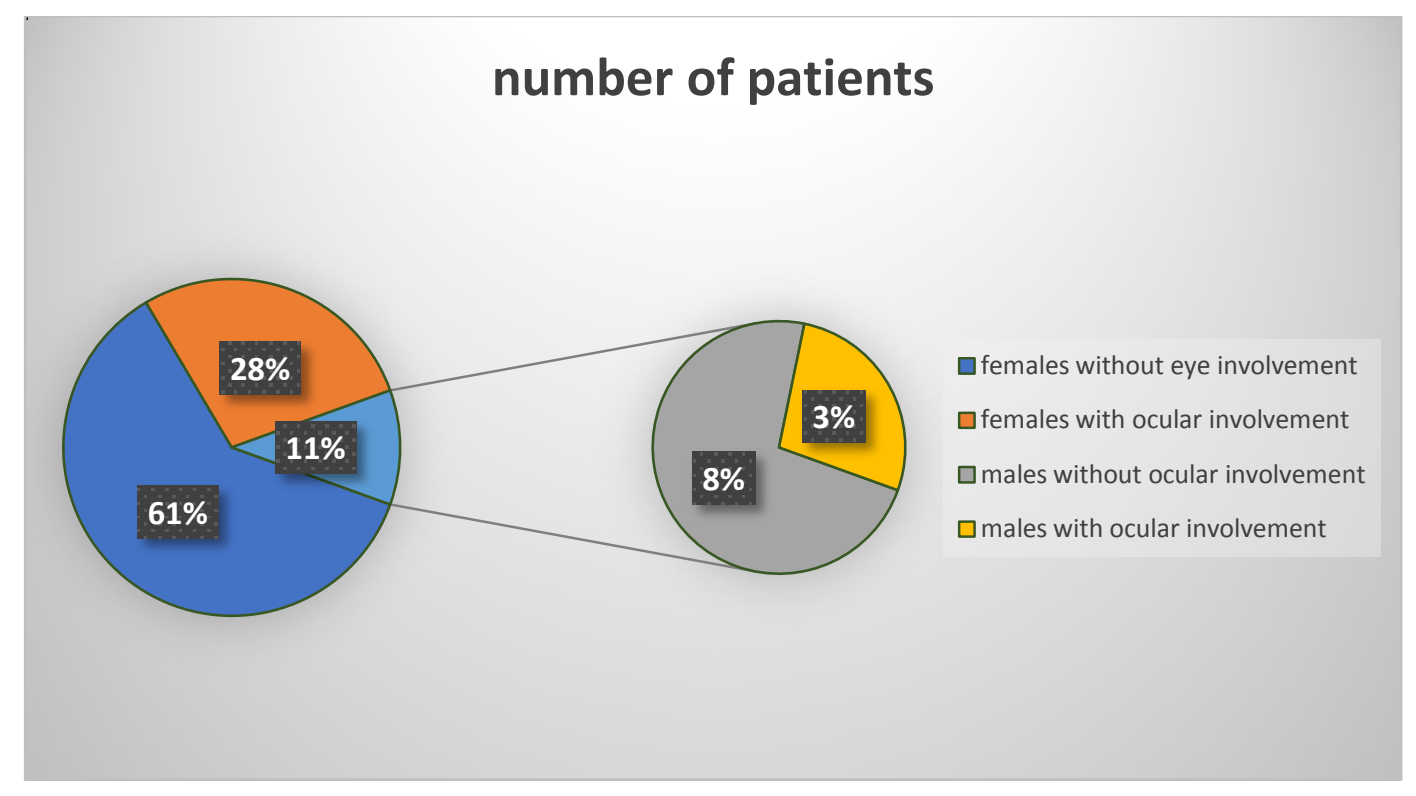

There was no significant difference in ocular involvement in both sexes ( $p$ value $>0.05$ ) Mean age of patients was 44. 28 \pm 9.72 years. The youngest patient was 19 years of age and the oldest 62 years.
Different ocular complaints and signs were recorded. The various symptoms with which the patients presented were as shown in figure 1.

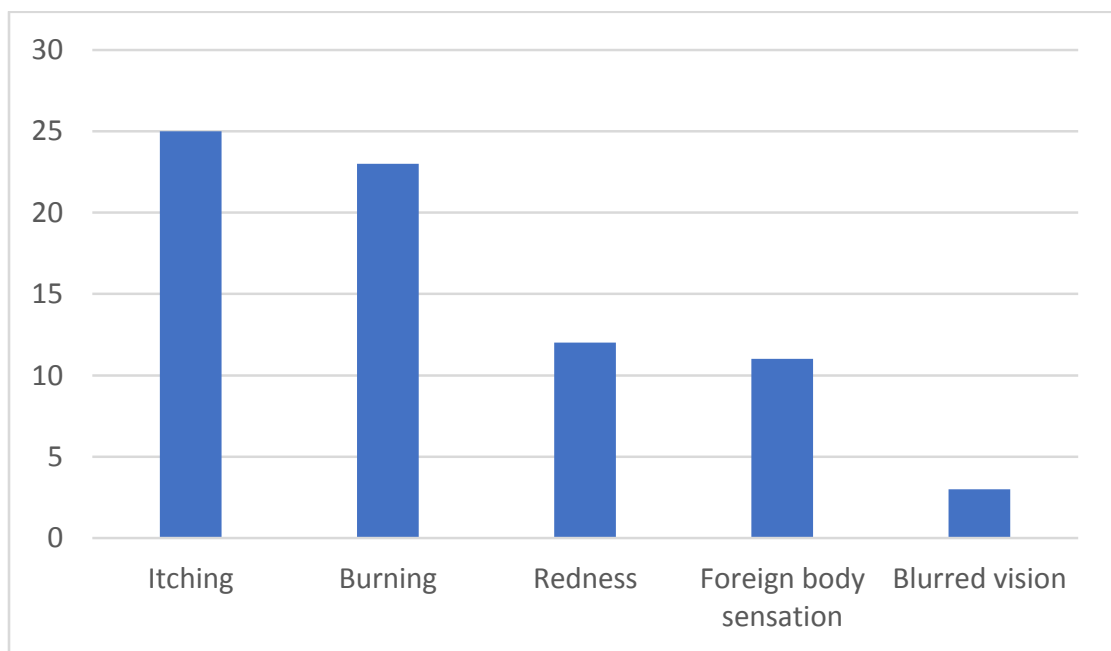

Figure 1: Showing the distribution of symptoms in patients of ocular rosacea

Maximum patients complained of itching (80.6\%), followed by burning (74.2\%) and redness $(38.7 \%)$ Eyelid signs were seen in all the 31 patients $(100 \%)$, conjunctival involvement was seen in
$64.5 \%$ patients and corneal involvement in 16.1 $\%$ patients Various eyelid signs were as shown in Figure 2 


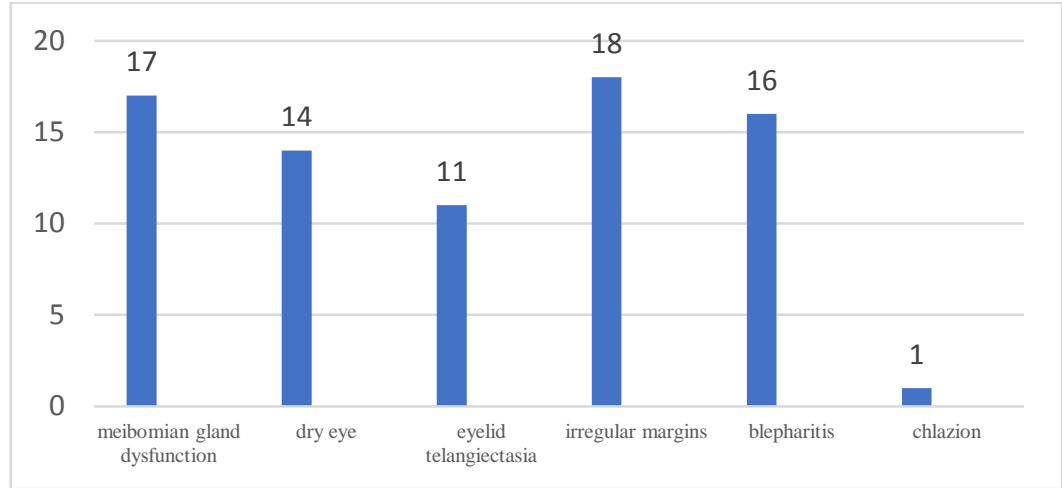

Figure 2 Showing eyelid involvement in ocular rosacea

Conjunctival involvement was seen in form of hyperaemia. Corneal involvement was seen in 5 patients (pannus formation was seen in two, corneal scarring .vascularization and thinning was seen in one patient (Figure 3). Inferior punctate keratitis was seen in 4 patients.

\section{Discussion}

Rosacea is a chronic inflammatory disorder manifesting as facial erythema ,flushing and telangiectasia with intermittent episodes of inflammation. In 2002, the National Rosacea Society Expert Committee classified rosacea into erythematotelangiectatic, papulopustular, phymatous and ocular type. ${ }^{[3]}$ The revised criteria as suggested by global rosacea Panel 2016 suggests that one diagnostic phenotype and two major criteria are required for diagnosis of rosacea. $^{[4]}$ Ocular rosacea was first described in African American patients in 1986. ${ }^{[5]}$ Ocular rosacea is seen to follow cutaneous involvement in $53 \%$ cases may precede in only 20 percent casesoccurs concurrently with cutaneous disease in $27 \%$ cases. ${ }^{[6]}$ In our study ocular involvement was seen in $31 \%$ of rosacea. Arman et al observed ocular involvement in $30 \%$ of rosacea patients. ${ }^{[7]}$ Ghanem et al observed ocular involvement in $26 \%$ of patients diagnosed as rosacea by a dermatologist. ${ }^{[8]}$

Pathogenesis of ocular rosacea is not well understood. Various factors are hypothesized to play a role in pathogenesis of ocular rosacea. Increased IL-1 $\alpha$, matrix metalloproteinases 8,9 tumor necrosis factor (TNF-alfa), overexpression of ICAM-1 (intercellular adhesion molecule 1) and HLA-DR, inflammatory in tear film suggest an inflammatory role of ocular rosacea. ${ }^{[9,10]}$ Presence of bacterial lipases lead to increased free fatty acids which leads to ocular rosacea. ${ }^{[1,12]}$ Increased expression of cathelecidins leads to increase in vascular endothelial growth factor. ${ }^{[13]}$ A decreased concentration of group IIA phospholipase A2 in tears is there. Meibomian gland inflammation leads to decreased and altered secretions from the gland leading to insufficient tear film formation blepharitis, keratoconjunctivitis sicca (dry eyes) and chalazia Mean age in our patients was was 44. 28 \pm 9.72 years (age range of 19-62 years) which was slightly less than other studies, where the mean age was $51-56$ years. ${ }^{[14,15]}$ The younger age of presentation in our study was probably due to overuse of steroids.

In our study there were more number of females $(90.3 \%)$ but the ocular involvement was seen in $31.4 \%$ of total females and $27.2 \%$ of total males. Equal ocular involvement has been observed by many researchers. ${ }^{[8,15]}$ Apkek observed eye involvement to be more in males $(1.3: 1)^{[14]}$

Careful history is very important in case of ocular rosacea as the symptoms are often non specific e.g., a foreign body, gritty, or dry sensation, burning, tearing, or redness. Frequently, the symptoms are out of proportion to the ocular signs. In our study the commonest ocular symptom was itching $(80.6 \%)$, followed by burning $(74.2 \%)$ and redness(38.7\%). Barker observed redness of eye in $(55.5 \%)$ and itching 
$(50.0 \%)$ to be the presenting complaint. ${ }^{[15]}$ Arman et al reported burning and stinging in all their patients of ocular rosacea followed by redness and photophobia. Itching, blurring of vision, watering, pain and swelling of eyes were the other presenting complaints. ${ }^{[7]}$

Ocular rosacea has wide range of eye signs varying from blepharitis to sight threatening conditions like corneal neovascularization, ulcer or perforation. ${ }^{[8,15,16]}$ There is inflammation of ocular surface and destruction of meibomian glands. Features of ocular surface involvement suggesting rosacea are lid margin telangiectases, interpalpebral conjunctival injection, spadeshaped infiltrates on the cornea, scleritis, and sclerokeratitis .Other non -specific features of ocular rosacea are chalazia, conjunctivitis, crusting on eyelashes, lid margin irregularity, and discontinuity of tear film. ${ }^{[8,15]}$ In our study lid margin irregularity $(58.06 \%)$ was commonest eye sign followed by meibomian gland dysfunction (MGD)(54.83\%). Other conjunctival signs were dry eye, blepharitis, eye lid telangiectasia and chlazion. Cornea was involved in 5(16.1\%) patients with one patient developing thinning and vascularization of cornea. Apkek also observed eyelid telangiectasia and irregularity to be commonest ( $81 \%$ ) ocular sign followed by MGD in $78 \%^{[14]}$ Ghanem et al observed much higher corneal involvement(47.2\%) with two patients developing glaucoma. ${ }^{[8]}$ Also they observed MGD in $85.2 \%$ and lid margin telangiectasia in $53.4 \%$ cases. OLtz et al reported corneal involvement in $33 \%$ patients. ${ }^{[2]}$ Apkek observed corneal ulceration in $5 \%$ cases $^{[14]}$ The lower rate of corneal involvement in ourstudy could be because maximum patients in our study were referred from dermatology departmentand presented early. Wise7 reported that the most common ocular signs in patients with rosacea from the ophthalmologic clinic were blepharitis (93\%), conjunctival hyperemia (80\%), and corneal vascularization and infiltrate $(67 \%) .{ }^{[16]}$ Jenkins et al. ${ }^{10}$ reported conjunctival hyperemia (86\%), telangiectasia of the lid margin $(63 \%)$, blepharitis
(47\%), and superficial punctate keratopathy $(41 \%)$.

Timely intervention can control the ophthalmic disease with appropriate therapeutic modalities including topical corticosteroids, topical cyclosporine and systemic doxycycline.

\section{Conclusion}

Ocular rosacea is an under diagnosed entity. Regular ophthalmologic examination should be done in every patient of rosacea. Timely diagnosis and treatment can prevent the sight threatening complications

\section{References}

1. Mi Vieira AC, Höfling-Lima AL, Mannis MJ. Ocular rosacea-a review. Arq Bras Oftalmol. 2012; 75: 363-9.

2. Oltz M, Check J. Rosacea and its ocular manifestations. Optometry 2011;82:92103.

3. Wilkin J, Dahl M, Detmar $M$, et al. Standard classification of rosacea: Report of the National Rosacea Society Expert Committee on the Classification and Staging of Rosacea. J Am Acad Dermatol 2002; 46:584

4. Gallo RL, Granstein RD, Kang S, et al. Standard classification and pathophysiology of rosacea: The 2017 update by the National Rosacea Society Expert Committee. J Am Acad Dermatol 2018; 78:148

5. Browning DJ, Rosenwasser G, Lugo M. "Ocular rosacea in blacks," American Journal of Ophthalmology 1986;101: 4414.

6. Borrie P. "Rosacea with special reference to its ocular manifestations," British Journal of Dermatology1953;. 65:458-63.

7. Arman A, Demirseren DD, Takmaz T. Treatment of ocular rosacea: comparative study of topical cyclosporine and oral doxycycline. Iny J Ophthalmol 2015;8:544-9. 
8. Ghanem VC, Mehra N, Wong S, Mannis MJ. The prevalence of ocular signs in acne rosacea: comparing patients from ophthalmology and dermatology clinics. Cornea 2003; 22:230.

9. Barton K, Dagoberto CM, Nava A, Pflugfelder SC: Inflammatory cytokines in the tears of patients with ocular rosacea. Ophthalmology 1997; 104:1868- 74.

10. Sobrin L, Liu Z, Monroy DC, et al.: Regulation of MMP-9activityinhumantear fluid and corneal epithelial culture supernatant. Invest Ophthalmol Vis Sci 2000, 41:1703-9.

11. Afonso AA, Sobrin, L, Monroy DC, Selzer M, Lokeshwar B, et al. "Tear fluid gelatinase $\mathrm{B}$ activity correlates with IL$1 \alpha$ concentration and fluorescein clearance in ocular rosacea," Investigative Ophthalmology and Visual Science 1999;40 : 2506-12.

12. Joanna S. Saade, Bachir Abiad, Jonathan Jan, Dana Saadeh, James P. McCulley, and Jeremy Bartley, "Ocular Rosacea Causing Corneal Melt in an African American Patient and a Hispanic Patient," Case Reports in Ophthalmological Medicine, vol. 2017, Article ID 2834031, 5 pages, 2017. https://doi.org/10.1155/2017/283403 1

13. Wladis EJ, Iglesias BV, Adam AP, Gosselin EJ. Molecular biologic assessment of cutaneous specimens of ocular rosacea. Ophthal Plast Reconstr Surg. 2012;28(4):246-50.

14. Akpek EK, Merchant A, Pinar V, Foster CS., "Ocular rosacea: Patient characteristics and followup," Ophthalmology 1997;104:18637.Starr PA, McDonald A. Oculocutaneous aspects of rosacea. Proc R Soc Med. 1969;62:9-11.
15. Bakar O, Demircay Z, Toker E, Cakı S. Ocular signs, symptoms and tear function tests of papulopustular rosacea patients receiving azithromycin. JEADV 2009; 23: 544-9.

16. Wise G. Ocular rosacea. Am J Ophthalmol. 1943;26:591-609.

17. Jenkins MA, Brown SI, Lempert SL, et al. Ocular rosacea. Am J Ophthalmol 1979;88:618-22. 